\title{
Nonrealizable Minimal Vertex Triangulations of Surfaces: Showing Nonrealizability Using Oriented Matroids and Satisfiability Solvers
}

\author{
Lars Schewe
}

Received: 17 January 2008 / Revised: 7 August 2009 / Accepted: 12 August 2009 /

Published online: 2 September 2009

(C) Springer Science+Business Media, LLC 2009

\begin{abstract}
We show that no minimal vertex triangulation of a closed, connected, orientable 2-manifold of genus 6 admits a polyhedral embedding in $\mathbb{R}^{3}$. We also provide examples of minimal vertex triangulations of closed, connected, orientable 2-manifolds of genus 5 that do not admit any polyhedral embeddings. Correcting a previous error in the literature, we construct the first infinite family of such nonrealizable triangulations of surfaces. These results were achieved by transforming the problem of finding suitable oriented matroids into a satisfiability problem. This method can be applied to other geometric realizability problems, e.g., for face lattices of polytopes.
\end{abstract}

Keywords Polyhedral surfaces $\cdot$ Embeddings $\cdot$ Oriented matroids $\cdot$ Satisfiability

Grünbaum conjectured [19, Exercise 13.2.3] that all triangulated surfaces (compact, orientable, connected, 2-dimensional manifolds without boundary) admit polyhedral embeddings, also called geometric realizations, in $\mathbb{R}^{3}$. This conjecture was shown to be false by Bokowski and Guedes de Oliveira [6]. They showed that one special triangulation with 12 vertices of a surface of genus 6 does not admit a polyhedral embedding in $\mathbb{R}^{3}$. Recently, Archdeacon, Bonnington, and Ellis-Monaghan [2] settled the case of genus 1 by showing that all triangulations of the torus admit a polyhedral embedding.

Triangulated surfaces with polyhedral embeddings may be quite complicated (for an introduction, see [35]). McMullen, Schulz, and Wills [26] constructed polyhedral embeddings of triangulated surfaces with $n$ vertices of genus $\Theta(n \log n)$. Neverthe-

This work is part of the $\mathrm{PhD}$ thesis of the author. The author was supported by a scholarship of the Deutsche Telekom Foundation.

L. Schewe (凶)

Fachbereich Mathematik, TU Darmstadt, Schlossgartenstrasse 7, 64289 Darmstadt, Germany

e-mail: schewe@mathematik.tu-darmstadt.de 
less, a gap remains: Jungerman and Ringel [22] showed that $n$ vertices suffice to triangulate a surface of genus $\Theta\left(n^{2}\right)$ by explicitly constructing such triangulations.

So, can we construct polyhedral embeddings of triangulated surfaces with few vertices? For 2-spheres the combinatorial bound is sharp; this is a consequence of Steinitz's Theorem [32]. Additionally, all vertex-minimal triangulations of surfaces up to genus 4 admit polyhedral embeddings: For genus 1, a construction was given by Császár [15], the case of genus 2 was solved by Lutz [24] and Bokowski [4], and the cases of genus 3 and 4 by Hougardy, Lutz, and Zelke [21]).

Our main result is that none of the vertex minimal triangulations of a surface of genus 6 admits a polyhedral embedding in $\mathbb{R}^{3}$. Moreover, three minimal triangulations of a surface of genus 5 do not admit such embeddings either. A small modification of one of the triangulations allows us to construct an infinite family of nonembeddable triangulated surfaces. For all results, we use an improved method to construct oriented matroids that are admissible for the surface in question. The method can also be applied to embedding problems for general simplicial complexes in arbitrary dimensions. A variant of the method allows us to also treat immersions of simplicial complexes. Using this variant, we can show for 58 of the 59 triangulations of a surface of genus 6 with 12 vertices that they cannot be immersed into $\mathbb{R}^{3}$. We are not able to settle the remaining case.

The new method we propose to generate oriented matroids reduces the generation problem to an instance of the satisfiability problem. Therefore, we can use well-tuned software, which speeds up the checking process immensely. As oriented matroids have been used to tackle other geometric realizability problems, our method gives more effective algorithms for these problems as well.

\section{Results}

Using the algorithm given below, it was possible to show the following theorems:

Theorem 1.1 No triangulation of a surface of genus 6 with 12 vertices admits a polyhedral embedding in $\mathbb{R}^{3}$.

The theorem is a consequence of the following proposition. A key step is the classification of combinatorial surfaces with 12 vertices of genus 6 by Altshuler, Bokowski, and Schuchert [1].

Proposition 1.2 None of the 59 combinatorial surfaces with 12 vertices of genus 6 admits an acyclic, uniform oriented matroid.

The situation is more difficult in the case of genus 5. To triangulate a surface of genus 5, we also need at least 12 vertices. The number of possible triangulations with 12 vertices, however, is much larger than in the case of genus 6: Lutz and Sulanke [33] showed that 751593 combinatorial triangulations exist. Therefore treating all cases algorithmically seems infeasible. Moreover, Hougardy, Lutz, and Zelke [21] showed that some of these triangulations can be embedded in $\mathbb{R}^{3}$. We were, nevertheless, able to find triangulations that cannot be embedded. 
Table 1 Triangulation ${ }^{2} 12_{1}^{1}$ of [23]

\begin{tabular}{rrrrrrrrrrrrrrrrrr}
\hline 1 & 2 & 3 & 1 & 2 & 12 & 1 & 3 & 6 & 1 & 4 & 8 & 1 & 4 & 11 & 1 & 5 & 9 \\
1 & 5 & 10 & 1 & 6 & 9 & 1 & 8 & 10 & 1 & 11 & 12 & 2 & 3 & 4 & 2 & 4 & 7 \\
2 & 5 & 9 & 2 & 5 & 12 & 2 & 6 & 10 & 2 & 6 & 11 & 2 & 7 & 10 & 2 & 9 & 11 \\
3 & 4 & 5 & 3 & 5 & 8 & 3 & 6 & 10 & 3 & 7 & 11 & 3 & 7 & 12 & 3 & 8 & 11 \\
3 & 10 & 12 & 4 & 5 & 6 & 4 & 6 & 9 & 4 & 7 & 11 & 4 & 8 & 12 & 4 & 9 & 12 \\
5 & 6 & 7 & 5 & 7 & 10 & 5 & 8 & 12 & 6 & 7 & 8 & 6 & 8 & 11 & 7 & 8 & 9 \\
7 & 9 & 12 & 8 & 9 & 10 & 9 & 10 & 11 & 10 & 11 & 12 & & & & & \\
\hline
\end{tabular}

Table 3 Triangulation ${ }^{2} 12_{6}^{1}$ of [23]
Table 2 Triangulation ${ }^{2} 12_{2}^{1}$ of [23]

\begin{tabular}{rrrrrrrrrrrrrrrrrr}
\hline 1 & 2 & 3 & 1 & 2 & 12 & 1 & 3 & 6 & 1 & 4 & 9 & 1 & 4 & 11 & 1 & 5 & 8 \\
1 & 5 & 9 & 1 & 6 & 10 & 1 & 8 & 10 & 1 & 11 & 12 & 2 & 3 & 4 & 2 & 4 & 7 \\
2 & 5 & 10 & 2 & 5 & 12 & 2 & 6 & 9 & 2 & 6 & 10 & 2 & 7 & 11 & 2 & 9 & 11 \\
3 & 4 & 5 & 3 & 5 & 8 & 3 & 6 & 11 & 3 & 7 & 10 & 3 & 7 & 11 & 3 & 8 & 12 \\
3 & 10 & 12 & 4 & 5 & 6 & 4 & 6 & 9 & 4 & 7 & 12 & 4 & 8 & 11 & 4 & 8 & 12 \\
5 & 6 & 7 & 5 & 7 & 10 & 5 & 9 & 12 & 6 & 7 & 8 & 6 & 8 & 11 & 7 & 8 & 9 \\
7 & 9 & 12 & 8 & 9 & 10 & 9 & 10 & 11 & 10 & 11 & 12 & & & & & \\
\hline
\end{tabular}

\begin{tabular}{rrrrrrrrrrrrrrrrrr}
\hline 1 & 2 & 4 & 1 & 2 & 6 & 1 & 3 & 6 & 1 & 3 & 12 & 1 & 4 & 11 & 1 & 5 & 9 \\
1 & 5 & 12 & 1 & 8 & 9 & 1 & 8 & 10 & 1 & 10 & 11 & 2 & 3 & 5 & 2 & 3 & 7 \\
2 & 4 & 7 & 2 & 5 & 12 & 2 & 6 & 10 & 2 & 9 & 10 & 2 & 9 & 11 & 2 & 11 & 12 \\
3 & 4 & 6 & 3 & 4 & 8 & 3 & 5 & 8 & 3 & 7 & 11 & 3 & 10 & 11 & 3 & 10 & 12 \\
4 & 5 & 7 & 4 & 5 & 9 & 4 & 6 & 9 & 4 & 8 & 12 & 4 & 11 & 12 & 5 & 6 & 8 \\
5 & 6 & 10 & 5 & 7 & 10 & 6 & 7 & 9 & 6 & 7 & 11 & 6 & 8 & 11 & 7 & 8 & 10 \\
7 & 8 & 12 & 7 & 9 & 12 & 8 & 9 & 11 & 9 & 10 & 12 & & & & & & \\
\hline
\end{tabular}

Theorem 1.3 At least three combinatorially distinct triangulations of a surface of genus 5 with 12 vertices do not admit a polyhedral embedding in $\mathbb{R}^{3}$.

More specifically, no admissible oriented matroids exist for the manifolds ${ }^{2} 12_{1}^{1}$, ${ }^{2} 12_{2}^{1}$, and ${ }^{2} 12_{6}^{1}$ described in the dissertation of Frank Lutz [23]. A facet description of these nonembeddable manifolds can be found in Tables 1,2, 3 .

Bokowski and Guedes de Oliveira [6] considered another question: Are there infinite families of surfaces of a fixed genus that cannot be realized? They also tried to construct such a family; we found, however, that their construction contained a flaw. Nevertheless, such families exist:

Theorem 1.4 For each genus $g \geq 5$, there exist infinite families of surfaces that have no polyhedral embedding in $\mathbb{R}^{3}$.

Bokowski and Guedes de Oliveira tried to construct such a family by taking a nonrealizable triangulation and removing a triangle such that the remaining complex stays nonrealizable. We found that the remaining complex given by Bokowski and Guedes de Oliveira admits chirotopes after all. Their argument for nonrealizability 
Table 4 Surface no. 1 of [1]

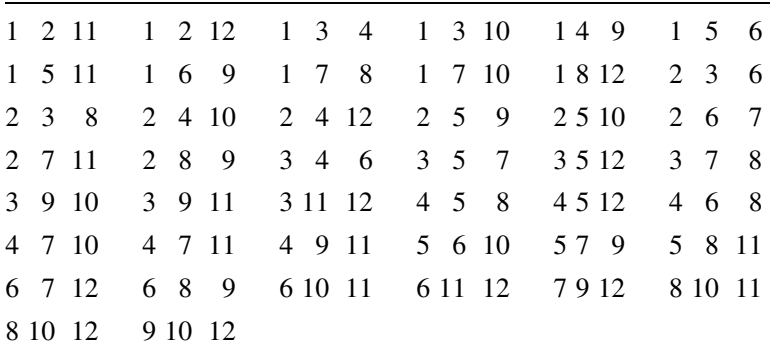

depends crucially on the symmetry of the surface to reduce the search space. However, the symmetry group of the complex in question is smaller than the symmetry group of the whole triangulation.

Still, our construction of the families in Theorem 1.4 uses the main idea of Bokowski and Guedes de Oliveira [6]. We take the connected sum of suitable surfaces: We can ensure that this connected sum is nonrealizable if one of the summands stays nonrealizable after the removal of the triangle along which we connect the summands. As we do not need to impose any conditions on the second summand, we can then construct surfaces of arbitrary genus $g$ as long as $g$ is greater than or equal to the genus of the first summand. By additionally adding triangulations of spheres with arbitrary numbers of vertices, we can construct the infinite families we are after. The construction is summarized in the following lemma. We omit the straightforward proof.

Lemma 1.5 Given two triangulations $\mathrm{S}$ and $\mathrm{T}$ of surfaces and a triangle $T \in \mathrm{S}$ such that $\mathrm{S} \backslash\{T\}$ cannot be polyhedrally embedded, there exists a triangulation $\mathrm{X}$ with $V(\mathrm{X})=V(\mathrm{~S})+V(\mathrm{~T})-3$ vertices of a surface of genus $g_{\mathrm{S}}+g_{\mathrm{T}}$ that cannot be polyhedrally embedded as well.

The following proposition shows that the conditions of the lemma can be satisfied and thus completes the proof of Theorem 1.4.

Proposition 1.6 (a) Let $\mathrm{O}$ be the surface ${ }^{2} 12_{1}^{1}$ (see Table 1 ), and let $\mathrm{M}:=\mathrm{O} \backslash$ $\{\{1,2,3\}\}$. Then $\mathrm{M}$ does not admit an acyclic uniform oriented matroid.

(b) Let $\mathrm{P}$ be the surface no. 1 in the enumeration of Altshuler and Bokowski [1] (see Table 4), and let $\mathrm{N}:=\mathrm{P} \backslash\{\{1,2,11\}\}$. Then $\mathrm{N}$ does not admit an acyclic uniform oriented matroid.

Our results depend on a method to generate oriented matroids, which we describe in the next section. We first give an overview before we deal with the technical details.

\section{The Main Algorithm}

We want to treat the embeddability problem algorithmically. An embedding is a map that assigns to each vertex of a simplicial complex coordinates in $\mathbb{R}^{n}$ such that no two vertex-disjoint simplices intersect each other nontrivially. This intersection condition 
can be expressed by a set of polynomials in the coordinates. However, we want to use a combinatorial approach. To do so, we need a combinatorial model of a point set in $\mathbb{R}^{n}$, which captures interesting properties (for instance, convexity). Oriented matroids are a good choice for this purpose. Examples of such applications can be found, for instance, in the book by Bokowski and Sturmfels [7]. The resulting combinatorial conditions will only provide a necessary condition for embeddability. However, they are easier to check algorithmically than algebraic conditions. In fact, the main algorithmic contribution of this article is the observation that these conditions can be expressed as an instance of the satisfiability problem and can, thus, be checked effectively.

\subsection{Simplicial Complexes and Their Embeddings}

We first need to fix the definitions for combinatorial surfaces and their embeddings. The most general object we look at is an abstract simplicial complex $\mathrm{K}$. It is defined over a finite set $V(\mathrm{~K})$ of vertices and consists of a set of simplices $\mathrm{K} \subseteq 2^{V(\mathrm{~K})}$ such that, for each $\Delta \subseteq \mathrm{K}$ and all $F \subseteq \Delta$, it holds that $F \in \mathrm{K}$. Now we want to embed such a complex into a $d$-dimensional space. For this, let $f: V(\mathrm{~K}) \rightarrow \mathbb{R}^{d}$ be an injective map. We can extend this map to map subsets of $V(\mathrm{~K})$ to subsets of $\mathbb{R}^{d}$ by setting $\bar{f}: \Delta \mapsto \operatorname{conv}\{f(v) \mid v \in \Delta\}$, where conv denotes the convex hull. We further want such a map to be nondegenerate in the sense that simplices do not get mapped to lower-dimensional simplices. For this, we have to require that the vertices of the geometric simplex $\bar{f}(\Delta)$, which is the image of $\Delta$ under $\bar{f}$, coincide with the images of the elements of $\Delta$ under $f$. Moreover, for $f$ to be an embedding of an abstract simplicial complex $\mathrm{K}$, we need to ensure that the images of two simplices have nontrivial intersection in $\mathbb{R}^{d}$ if and only if the simplices have a nontrivial intersection in the complex K. Combined, the definition of an embedding is as follows:

Definition 2.1 (Embedding) Given a simplicial complex $\mathrm{K}$ and a map $f: V(\mathrm{~K}) \rightarrow$ $\mathbb{R}^{d}$, we say that the mapping $f$ induces a polyhedral embedding if it has the following two properties:

- For all $\Delta \in \mathrm{K}$, it holds that $\{f(v) \mid v \in \Delta\}=\operatorname{vert}(\bar{f}(\Delta))$.

- For all $\Delta_{1}, \Delta_{2} \in \mathrm{K}$ with $\Delta_{1} \cap \Delta_{2}=\emptyset$, it holds that $\bar{f}\left(\Delta_{1}\right) \cap \bar{f}\left(\Delta_{2}\right)=\emptyset$.

Polyhedral embeddings of triangulated surfaces are "nice" in the following sense: we can perturb the vertices by a small amount without creating any intersections of the simplices. Therefore, we may assume that the points lie in general position, that is, no four points lie on a common plane. We can therefore restrict the set of possible embeddings to polyhedral embeddings in general position.

As mentioned above, our goal is to construct a combinatorial condition for embeddability. We will first give a criterion to decide whether two simplices intersect. To test for the intersection of simplices, we will use a test based on Radon's theorem [31] (cf. [29]). We need a special case of the theorem: Given a set $E$ of $(d+2)$ points in $\mathbb{R}^{d}$ in general position, there exists a unique partition $(U, V)$ of $E$ into two sets such that conv $U \cap \operatorname{conv} V \neq \emptyset$. Such a partition is called a Radon partition. As a consequence of this theorem, we obtain the following criterion: Given a triangle $T$ and a 
line segment $L$ in $\mathbb{R}^{3}$ with the point set vert $T \cup \operatorname{vert} L$ in general position, $T$ and $L$ intersect if and only if the Radon partition of the point set vert $T \cup \operatorname{vert} L$ is given by (vert $T$, vert $L$ ). This condition can be encoded using a combinatorial abstraction: oriented matroids.

\subsection{Oriented Matroids}

The following discussion of oriented matroids is extremely brief; we recommend the monograph [3], especially Sect. 3.5 for the missing details. We only consider uniform oriented matroids, which (in the realizable case) correspond to point sets in general position. We will use the following axioms for oriented matroids:

Definition 2.2 Let $E$ be a finite set, $r \in \mathbb{N}$ with $|E| \geq r$, and $\chi: E^{r} \rightarrow\{-1,+1\}$. We call $\mathcal{M}=(E, \chi)$ a uniform oriented matroid of rank $r$ if the following conditions are satisfied:

(B1) The mapping $\chi$ is alternating, i.e., for all permutations $\pi$ of $\{1, \ldots, r\}$, it holds that $\chi\left(e_{1}, \ldots, e_{r}\right)=\operatorname{sgn}(\pi) \chi\left(e_{\pi(1)}, \ldots, e_{\pi(r)}\right)$.

(B2) For all $\sigma \in\left(\begin{array}{c}E \\ r-2\end{array}\right)$ and all subsets $\left\{x_{1}, \ldots, x_{4}\right\} \subseteq E \backslash \sigma$, the following holds:

$$
\begin{aligned}
\{-1,+1\} \subseteq & \left\{\chi\left(\sigma, x_{1}, x_{2}\right) \chi\left(\sigma, x_{3}, x_{4}\right),\right. \\
& \left.-\chi\left(\sigma, x_{1}, x_{3}\right) \chi\left(\sigma, x_{2}, x_{4}\right), \chi\left(\sigma, x_{1}, x_{4}\right) \chi\left(\sigma, x_{2}, x_{3}\right)\right\} .
\end{aligned}
$$

Remark 2.3 The mapping $\chi$ is called the chirotope of the oriented matroid. In the more general case where $\chi$ may also take the value zero, the oriented matroid is called nonuniform. However, as the axiom (B2) gets more complicated in that case, we restrict ourselves to the uniform case. For normalization purposes, we will, additionally, assume that we are given a total order of $E$.

As a first consequence of these axioms, we can restrict our attention to the values that $\chi$ attains on the ordered $r$-subsets of $E$. The other values are then determined by (B1).

Given a set of points $E$ in $\mathbb{R}^{d}$, we can construct a chirotope the following way: Express the points in homogeneous coordinates using the map $v \mapsto \widehat{v}=\left(\begin{array}{l}1 \\ v\end{array}\right)$ and then set for any $e_{1}, \ldots, e_{d+1} \in E, \chi\left(e_{1}, \ldots, e_{d+1}\right)=\operatorname{sgn} \operatorname{det}\left(\widehat{e}_{1}, \ldots, \widehat{e}_{d+1}\right)$. We call $E$ the realization of the oriented matroid $(E, \chi)$. We call an oriented matroid realizable if it has a realization. The rank of the oriented matroid $(E, \chi)$ constructed from $E \subset \mathbb{R}^{d}$ in this way is $d+1$. It is not obvious that every chirotope we construct this way satisfies condition (B2). Condition (B2) is, in fact, a combinatorial variant of the GrassmannPlücker relations, a classical determinant identity (for definitions and proofs, we refer to [3, Sects. 2.4 and 3.6]). Therefore the construction above, in fact, yields an oriented matroid.

To detect intersections we will use the oriented matroid analogue of Radon partitions: the circuits of the oriented matroid. For "ordinary," i.e., nonoriented, matroids, the circuits are the minimally dependent sets. As we are looking at uniform oriented matroids, all $(r+1)$-element sets are dependent, and all $r$-element sets independent. 
Thus, in the realizable case every $(d+2)$-element set is a circuit. The circuits of oriented matroids is a circuit of the underlying matroid together with a signature, i.e., a function that assigns to each element of the circuit a sign: In the realizable case we can use these signs to read of the Radon partition of the underlying circuit.

Given a uniform oriented matroid $\mathcal{M}=(E, \chi)$, the circuit signatures of $\mathcal{M}$ can be computed from the chirotope: Let $\underline{C}=\left(c_{1}, \ldots, c_{r+1}\right)$ (where the $c_{i}$ are taken from $E$, and $c_{1}<\cdots<c_{r+1}$ holds) be an unoriented circuit. Then there exist two possible signatures $s^{+}$and $s^{-}$of $\underline{C}$ in $\mathcal{M}$, and they are given by $s_{i}^{+}=$ $(-1)^{i} \chi\left(c_{1}, \ldots, c_{i-1}, c_{i+1} \ldots, c_{r+1}\right)$ and $s^{-}=-s^{+}$(see also [3, Lemma 3.5.7]). We denote a circuit $C$ as $C=\left(s_{1} c_{1}, \ldots, s_{r+1} c_{r+1}\right)$, where the underlying unoriented circuit is $\underline{C}=\left(c_{1}, \ldots, c_{r+1}\right)$, and $s_{i}$ denotes the sign of element $c_{i}$.

The general intersection condition for two simplices is therefore given as follows: If $\Delta_{1}$ and $\Delta_{2}$ are simplices such that $\Delta_{1} \cap \Delta_{2}=\emptyset$, they intersect in a realizable oriented matroid $\mathcal{M}$ if and only if the set $\Delta_{1} \cup \Delta_{2}$ contains a circuit $C=\left(s_{1} c_{1}, \ldots, s_{r+1} c_{r+1}\right)$ of $\mathcal{M}$ such that

$$
C^{+}=\left\{c_{i} \mid s_{i}=+1, i \in\{1, \ldots, r+1\}\right\} \subseteq \Delta_{1}
$$

and

$$
C^{-}=\left\{c_{i} \mid s_{i}=-1, i \in\{1, \ldots, r+1\}\right\} \subseteq \Delta_{2} .
$$

We say that an oriented matroid $\mathcal{M}=(E, \chi)$ is admissible for a simplicial complex $\mathrm{K}$ if $E=V(\mathrm{~K})$ and for all $\Delta_{1}, \Delta_{2} \in \mathrm{K}$ with $\Delta_{1} \cap \Delta_{2}=\emptyset$, there does not exist any circuit $C$ such that $C^{+} \subseteq \Delta_{1}$ and $C^{-} \subseteq \Delta_{2}$. If we consider only uniform oriented matroids of rank 4 and our simplices are faces of a surface, we only need to consider the case that $\Delta_{1}$ is a triangle and $\Delta_{2}$ is an edge. Additionally, we use a known fact about realizable oriented matroids: no circuit of such an oriented matroid is totally positive. Oriented matroids with this property are called acyclic.

In conclusion, given a simplicial complex $\mathrm{K}$, we can deduce that $\mathrm{K}$ cannot be embedded in $\mathbb{R}^{d}$ if $\mathrm{K}$ does not admit any acyclic, uniform oriented matroid of rank $d+1$. We will now check for this condition by constructing an instance of SAT that is satisfiable if and only if such an oriented matroid exists. The construction consists of two main parts: the encoding of the oriented matroid axioms, mainly condition (B2), and the encoding of the "forbidden" circuits.

\subsection{SAT}

Before we give our transformation, we first fix our notation for instances of SAT.

Take a Boolean function $\Phi:\{0,1\}^{n} \rightarrow\{0,1\}$, where 0 stands for false and 1 for true. We call the elements of $\{0,1\}^{n}$ valuations. A valuation $v \in\{0,1\}^{n}$ is satisfying if $\Phi(v)=1$.

An instance of SAT consists of a Boolean function given in conjunctive normal form $(\mathrm{CNF})$. That is, given the variables $p_{1}, \ldots, p_{n}$, the function $\Phi$ is of the form $\Phi(p)=\bigwedge_{i=0}^{m} C_{i}$ where the $C_{i}$ are of the form $C_{i}=\bigvee_{j \in I_{i}} p_{j} \vee \bigvee_{j \in \bar{I}_{i}} \bar{p}_{j}$. An SAT solver answers the question whether $\Phi$ is satisfiable, i.e., whether there exists a satisfying valuation. In that case it returns a valuation $v$ such that $\Phi(v)=1$. 
The following observation goes back to Peirce [28]. It gives us a way to write an arbitrary Boolean function in CNF.

Lemma 2.4 Let $\Phi$ be a Boolean function $\Phi:\{0,1\}^{n} \rightarrow\{0,1\}$. Then we can write $\Phi$ as

$$
\Phi(x)=\bigwedge_{\substack{\sigma \in\{0,1\}^{n} \\ \neg \Phi(\sigma)}}\left(\bigvee_{i \in\left\{j \mid \sigma_{j}=1\right\}} \overline{x_{i}}\right) \vee\left(\bigvee_{i \in\left\{j \mid \sigma_{j}=0\right\}} x_{i}\right)
$$

\subsection{Encoding}

We are now ready to give the transformation of our problem: Given a simplicial complex $\mathrm{K}$ with vertex set $E$ and a dimension $d$, we want to decide whether there exists an acyclic, uniform oriented matroid of rank $r=d+1$ on $n=|E|$ points that is admissible for $\mathrm{K}$.

To encode the chirotope, we introduce a variable for each ordered $r$-subset $B$ of $E$, which we denote by $[B]$. Given a valuation $v$ defined on these variables, we construct a chirotope $\chi_{v}$ as follows: If $v([B])=1$, we set $\chi_{v}(B)=+1$, and if $v([B])=0$, then we set $\chi_{v}(B)=-1$.

We start by encoding the oriented matroid axioms. We do not deal explicitly with the axiom (B1) as we only need to fix the signs for the ordered subsets. Given ordered $r$-subsets $\alpha, \beta, \gamma, \delta, \epsilon, \zeta$ of $E$, we define the following clauses:

$$
\begin{aligned}
& G P(\alpha, \beta, \gamma, \delta, \epsilon, \zeta)=(\neg[\alpha] \vee \neg[\beta] \vee \neg[\gamma] \vee[\delta] \vee \neg[\epsilon] \vee \neg[\zeta]) \\
& \wedge(\neg[\alpha] \vee \neg[\beta] \vee \neg[\gamma] \vee[\delta] \vee[\epsilon] \vee[\zeta]) \\
& \wedge(\neg[\alpha] \vee \neg[\beta] \vee[\gamma] \vee \neg[\delta] \vee \neg[\epsilon] \vee \neg[\zeta]) \\
& \wedge(\neg[\alpha] \vee \neg[\beta] \vee[\gamma] \vee \neg[\delta] \vee[\epsilon] \vee[\zeta]) \\
& \wedge(\neg[\alpha] \vee[\beta] \vee \neg[\gamma] \vee \neg[\delta] \vee \neg[\epsilon] \vee[\zeta]) \\
& \wedge(\neg[\alpha] \vee[\beta] \vee \neg[\gamma] \vee \neg[\delta] \vee[\epsilon] \vee \neg[\zeta]) \\
& \wedge(\neg[\alpha] \vee[\beta] \vee[\gamma] \vee[\delta] \vee \neg[\epsilon] \vee[\zeta]) \\
& \wedge(\neg[\alpha] \vee[\beta] \vee[\gamma] \vee[\delta] \vee[\epsilon] \vee \neg[\zeta]) \\
& \wedge([\alpha] \vee \neg[\beta] \vee \neg[\gamma] \vee \neg[\delta] \vee \neg[\epsilon] \vee[\zeta]) \\
& \wedge([\alpha] \vee \neg[\beta] \vee \neg[\gamma] \vee \neg[\delta] \vee[\epsilon] \vee \neg[\zeta]) \\
& \wedge([\alpha] \vee \neg[\beta] \vee[\gamma] \vee[\delta] \vee \neg[\epsilon] \vee[\zeta]) \\
& \wedge([\alpha] \vee \neg[\beta] \vee[\gamma] \vee[\delta] \vee[\epsilon] \vee \neg[\zeta]) \\
& \wedge([\alpha] \vee[\beta] \vee \neg[\gamma] \vee[\delta] \vee \neg[\epsilon] \vee \neg[\zeta]) \\
& \wedge([\alpha] \vee[\beta] \vee \neg[\gamma] \vee[\delta] \vee[\epsilon] \vee[\zeta]) \\
& \wedge([\alpha] \vee[\beta] \vee[\gamma] \vee \neg[\delta] \vee \neg[\epsilon] \vee \neg[\zeta]) \\
& \wedge([\alpha] \vee[\beta] \vee[\gamma] \vee \neg[\delta] \vee[\epsilon] \vee[\zeta]) \text {. }
\end{aligned}
$$


The following proposition is a direct consequence of Lemma 2.4 and allows us to deal with axiom (B2).

Proposition 2.5 Let $\alpha, \beta, \gamma, \delta, \epsilon, \zeta$ be ordered $r$-subsets of $E, v \in\{0,1\}^{\left(\frac{|E|}{r}\right)}$, and $\chi_{v}$ defined as above. Then the following two conditions are equivalent:

1. $\left\{\chi_{v}(\alpha) \chi_{v}(\beta),-\chi_{v}(\gamma) \chi_{v}(\delta), \chi_{v}(\epsilon) \chi_{v}(\zeta)\right\} \supseteq\{+1,-1\}$

2. $v$ satisfies $G P(\alpha, \beta, \gamma, \delta, \epsilon, \zeta)$ as defined above.

So, each of the three-term Grassmann-Plücker relations, i.e., the conditions of axiom (B2), is encoded with 16 clauses with 6 literals each. As we have $\left(\begin{array}{c}n \\ r-2\end{array}\right)\left(\begin{array}{c}n-r+2 \\ 4\end{array}\right)$ different conditions from (B2) to consider, we get $16\left(\begin{array}{c}n \\ r-2\end{array}\right)\left(\begin{array}{c}n-r+2 \\ 4\end{array}\right)$ many clauses of length 6 in our resulting SAT instance. These clauses guarantee the property that each satisfiable valuation of the instance will correspond to a chirotope.

To complete the model we need a condition that excludes all oriented matroids that have a given circuit signature. As a special case, we want to exclude all oriented matroids that are not acyclic. The following proposition gives the exact condition; again the proposition follows directly from Lemma 2.4 :

Proposition 2.6 Let $\mathcal{M}=(E, \chi)$ be a uniform oriented matroid of rank $r, v$ the corresponding valuation, and $C=\left(s_{1} c_{1}, \ldots, s_{r+1} c_{r+1}\right)$ be a signed $(r+1)$-tuple (where for all $i \in\{1, \ldots, r+1\}, s_{i} \in\{+1,-1\}, c_{i} \in E$, and furthermore all $c_{i}$ are distinct). Then $C$ is not a circuit of $\chi$ if and only if $v$ satisfies $\Gamma(C)$ :

$$
\begin{aligned}
\Gamma(C)= & \bigwedge_{i \in I^{+}}\left[c_{1}, \ldots, c_{i-1}, c_{i+1}, \ldots, c_{r+1}\right] \\
& \wedge \bigwedge_{i \in I^{-}} \neg\left[c_{1}, \ldots, c_{i-1}, c_{i+1}, \ldots, c_{r+1}\right] \\
& \vee \bigwedge_{i \in I^{+}} \neg\left[c_{1}, \ldots, c_{i-1}, c_{i+1}, \ldots, c_{r+1}\right] \\
& \wedge \bigwedge_{i \in I^{-}}\left[c_{1}, \ldots, c_{i-1}, c_{i+1}, \ldots, c_{r+1}\right], \\
I^{+}= & \left\{i \mid(-1)^{i} s_{i}=+1\right\}, \\
I^{-}= & \left\{i \mid(-1)^{i} s_{i}=-1\right\} .
\end{aligned}
$$

Thus, we add, for every forbidden circuit, two clauses consisting of $r+1$ literals each. With these clauses, we have completed our SAT-instance. In the next section we will see how this gives us an effective way to solve our problem. If we want to use this method to treat other realizability problems, other restrictions are of interest. In the case of the algorithmic Steinitz problem, i.e., whether a lattice is a face lattice of 
a convex polytope, we need to generate oriented matroids with prescribed cocircuits. The necessary clauses can be derived in the same manner as described above.

\section{Implementation}

We wrote a Haskell [30] program that does the translation described in the preceding section. We then used the SAT-solvers ZChaff [25] and Minisat [16] to solve the resulting SAT instances. As input data in the case of genus 6, we used the abstract simplicial complexes as given in the article by Altshuler et al. [1]. To ensure that the simplicial complexes in our input files are combinatorial surfaces of genus 6 , we used the Polymake [18] system. The same checks were also used on the complexes taken from the dissertation of Lutz [23].

We tested our programs on known examples. We computed all chirotopes that are admissible for the Möbius torus. We found 2772 chirotopes (in less than 20 seconds), the same number found by Bokowski and Eggert [5]. Furthermore, we tested all triangulated surfaces with up to 9 vertices (including the nonorientable ones). In that case our program found out that all orientable surfaces admitted chirotopes, whereas the nonorientable ones did not. Additionally, we used our program to find chirotopes for all 821 minimal vertex triangulations of an orientable surface of genus 4 as classified by Lutz and Sulanke [33].

Several software packages that generate oriented matroids already exist (for instance, $[6,11,17,20])$. These packages use one of two different approaches: The programs by Bokowski and Guedes de Oliveira and by Finschi construct oriented matroids by using single-element extensions, whereas the other programs try to construct the oriented matroids globally by filling in the chirotopes. Our approach is of the second type. This type of approach was also used by Bremner and by Gugisch. Both have implemented software that uses a backtracking algorithm to set the values of a partial chirotope. We want to mention that David Bremner (personal communication) has also used a similar idea as presented here and modeled the axioms given above as a $0 / 1$ integer program. However, his benchmark results showed that his backtracking program MPC [11] is faster than this integer-programming-based approach in the instances he looked at.

To give an impression of the efficiency of our program, we state some running times: For the genus 6 examples, the transformation took approximately 30 seconds per instance. Solving the SAT instances took between 22 and 98 minutes. All times were taken on a machine with two Pentium III processors $(1 \mathrm{GHz})$ and $2 \mathrm{~GB}$ RAM. For all computations, only one processor was used. These results show that our program is much faster than the programs of Bokowski and Guedes de Oliveira [6].

One of the advantages of our method lies in the fact that one can use a variety of SAT solvers to check the results. Many SAT solvers allow the possibility to give a "proof" that an instance is unsatisfiable. They output how to derive a contradiction from the given input. However, this does not improve our situation: the proofs generated this way are so large that they can only be checked with the help of a computer. The construction of the SAT-instance from the simplicial complex is simple enough to be checked by hand. Future advances in the development of proof assistants might make it possible to give a full formal verification of our results. 


\section{Immersions}

We have seen that we cannot hope to find embeddings for all triangulations of orientable surfaces. However, one could hope for weaker results. Nonorientable surfaces, for instance, cannot be embedded for topological reasons. Here one tries instead to find immersions of these surfaces. Both positive and negative results are known for the problem of finding immersions of triangulations of nonorientable surfaces $[10,14]$. We, however, will only deal with the 59 triangulations of the (orientable) surface of genus 6 with 12 vertices. The definition of an immersion is as follows:

Definition 4.1 (Immersion) Given a triangulation $\mathbf{K}$ of a surface and a map $f$ : $V(\mathrm{~K}) \rightarrow \mathbb{R}^{d}$, we say that the mapping $f$ induces an immersion if it has the following two properties:

- For all $\Delta \in \mathrm{K}$, it holds that $\{f(v) \mid v \in \Delta\}=\operatorname{vert}(\bar{f}(\Delta))$.

- For all simplices $\Delta_{1}, \Delta_{2}$ in the star of a vertex $v \in V(\mathrm{~K})$ with $\Delta_{1} \cap \Delta_{2}=\emptyset$, it holds that $\bar{f}\left(\Delta_{1}\right) \cap \bar{f}\left(\Delta_{2}\right)=\emptyset$.

Remark 4.2 The star of a vertex is the inclusion minimal simplicial complex that contains all faces that contain the given vertex.

The definition of an immersion directly leads to an adaptation of the definition of an admissible oriented matroid. We say that an oriented matroid $\mathcal{M}=(E, \chi)$ is admissible with respect to an immersion of a simplicial complex $\mathrm{K}$ if $E=V(\mathrm{~K})$, and for all vertices $v \in V(\mathrm{~K})$ and simplices $\Delta_{1}, \Delta_{2} \in \operatorname{star}(v)$ with $\Delta_{1} \cap \Delta_{2}=\emptyset$, there does not exist any circuit $C$ such that $C^{+} \subseteq \Delta_{1}$ and $C^{-} \subseteq \Delta_{2}$.

Using a suitably modified version of the algorithm above (we just need to test fewer possible intersections), we can show that all but one of the 59 surfaces of genus 6 do not admit an oriented matroid that is admissible with respect to an immersion of that surface. The exception is surface number 15 (again using the numbering scheme used by Altshuler et al. [1]). Here we found many oriented matroids that are admissible. However, we were not able to find geometric realizations of one of these oriented matroids.

\section{Conclusion}

Our results give additional insight into the properties of minimal vertex triangulations of surfaces. Still, the main problems remain: How can we characterize nonrealizability? Do all triangulated surfaces of small genus (say $g \leq 4$ ) admit a polyhedral embedding?

The infinite class of nonrealizable surfaces given above suggests that there will be no easy answer to the first question. For genus 5 and 6, we can construct nonrealizable triangulations for any number of vertices. However, one of the main obstacles for tackling the question is the lack of good construction methods for "interesting" combinatorial surfaces. Such surfaces might, for instance, have an easily verifiable 
obstruction to embeddability. The linking number arguments of Brehm [9] might yield such an obstruction. However, no such example seems to be known in the case of a closed surface.

The method we used is interesting in its own right. It helps tremendously in the study of small examples. In turn, we hope that the small examples given here will help in the solution of the general problem. One major drawback remains: If we find oriented matroids in the course of our search, we cannot effectively use this information, because the realization problem for oriented matroids is difficult as well. The set of all realizations of a given oriented matroid can be described as the set of real solutions of a system of polynomial equalities and inequalities with integer coefficients. In fact, the realization problem for oriented matroids is as hard as deciding whether an arbitrary semi-algebraic set defined with integral coefficients is nonempty over the real numbers (cf. [3, Sect. 8.4]). Thus, one approach for finding a realization is to use tools from real algebraic geometry to decide the realizability of an oriented matroid. However, the polynomial systems we obtain from the realization problem for rank 4 oriented matroids with 12 elements are by far too large to be tackled with the known algorithms for deciding the nonemptiness of semi-algebraic sets. So far, only heuristic methods seem to be applicable (cf. [4]).

As an open problem remains the question how strong oriented matroid methods are compared to the methods described by Novik [27] and Timmreck [34]. We conjecture that the method outlined in this article is at least as strong, taken in the following sense: For each instance where the integer program proposed by Timmreck is infeasible, no admissible oriented matroid exists. We are led to this conjecture by the result of Carvalho and Guedes de Oliveira [13]. They showed that the linking number arguments given by Brehm as incorporated by Timmreck hold also in the setting of oriented matroids. This means that these arguments are subsumed by the oriented matroid technique. However, the combined strength of Novik's condition and the linking number condition is unknown.

The approach used in this article can be applied to other geometric problems. It has already been used to treat realizability of point-line configurations [8] and to bound the diameter of convex polytopes for certain parameters [12]. We hope that this technique proves itself to be a useful building block in these and other applications.

Acknowledgements I would like to thank Jürgen Bokowski for the advice and encouragement received while undertaking this work. I had several helpful discussions with a number of people: I like to mention Peter Lietz, Frank Lutz (who also generously provided me lots of interesting examples for testing my software), and Dagmar Timmreck. The comments of Sven Herrmann and an anonymous referee helped to improve the article. Thanks also to Michael Joswig who gave valuable comments on an earlier draft.

\section{References}

1. Altshuler, A., Bokowski, J., Schuchert, P.: Neighborly 2-manifolds with 12 vertices. J. Comb. Theory, Ser. A 75(1), 148-162 (1996)

2. Archdeacon, D., Bonnington, C.P., Ellis-Monaghan, J.A.: How to exhibit toroidal maps in space. Discrete Comput. Geom. 38(3), 573-594 (2007)

3. Björner, A., Las Vergnas, M., Sturmfels, B., White, N., Ziegler, G.M.: Oriented Matroids, 2nd edn. Encyclopedia of Mathematics and its Applications, vol. 46. Cambridge University Press, Cambridge (1999) 
4. Bokowski, J.: On heuristic methods of finding realizations of surfaces. In: Bobenko, A.I., Schröder, P., Sullivan, J.M., Ziegler, G.M. (eds.) Discrete Differential Geometry. Oberwolfach Seminars, vol. 38, pp. 255-260. Birkhäuser, Basel (2008)

5. Bokowski, J., Eggert, A.: All realizations of Möbius' torus with seven vertices. Struct. Topol. 17, 59-78 (1991)

6. Bokowski, J., Guedes de Oliveira, A.: On the generation of oriented matroids. Discrete Comput. Geom. 24(2-3), 197-208 (2000)

7. Bokowski, J., Sturmfels, B.: Computational Synthetic Geometry. Lecture Notes in Mathematics, vol. 1355. Springer, Berlin (1989)

8. Bokowski, J., Grünbaum, B., Schewe, L.: Topological configurations $\left(n_{4}\right)$ exist for all $n \geq 17$. Eur. J. Comb. (2009). doi:10.1016/j.ejc.2008.12.008, posted on January 13, 2009 (to appear in print)

9. Brehm, U.: A nonpolyhedral triangulated Möbius strip. Proc. Am. Math. Soc. 89(3), 519-522 (1983)

10. Brehm, U.: How to build minimal polyhedral models of the Boy surface. Math. Intell. 12(4), 51-56 (1990)

11. Bremner, D.: MPC-Matroid Polytope Completion, May 2008. Available at http://www.cs.unb.ca/ profs/bremner/mpc/

12. Bremner, D., Schewe, L.: Edge-graph diameter bounds for convex polytopes with few facets. Preprint (2008). arXiv:0809.0915

13. Carvalho, P., Guedes de Oliveira, A.: Intersection and linking numbers in oriented matroids. Discrete Comput. Geom. 31(2), 305-321 (2004)

14. Cervone, D.P.: Vertex-minimal simplicial immersions of the Klein bottle in three space. Geom. Dedic. 50(2), 117-141 (1994)

15. Császár, Á.: A polyhedron without diagonals. Acta Univ. Szeged. Sect. Sci. Math. 13, 140-142 (1949)

16. Eén, N., Sörensson, N.: An extensible SAT-solver. In: Giunchiglia, E., Tacchella, A. (eds.) SAT, 2003. Lecture Notes in Computer Science, vol. 2919, pp. 502-518. Springer, Berlin (2004)

17. Finschi, L., Fukuda, K.: Generation of oriented matroids-a graph theoretical approach. Discrete Comput. Geom. 27(1), 117-136 (2002)

18. Gawrilow, E., Joswig, M.: Polymake: a framework for analyzing convex polytopes. In: Kalai, G., Ziegler, G.M. (eds.) Polytopes-Combinatorics and Computation, pp. 43-74. Birkhäuser, Basel (2000)

19. Grünbaum, B.: Convex Polytopes, 2nd edn. Graduate Texts in Mathematics, vol. 221. Springer, New York (2003). Prepared and with a preface by V. Kaibel, V. Klee and G.M. Ziegler

20. Gugisch, R.: Konstruktion von Isomorphieklassen orientierter Matroide. Dissertation, Universitát Bayreuth (2005) (In German)

21. Hougardy, S., Lutz, F.H., Zelke, M.: Surface realization with the intersection edge functional. Exp. Math. to appear. Available at arXiv:math.MG/0608538

22. Jungerman, M., Ringel, G.: Minimal triangulations on orientable surfaces. Acta Math. 145(1-2), 121154 (1980)

23. Lutz, F.H.: Triangulated Manifolds with few Vertices and Vertex-Transitive Group Actions. Shaker, Aachen (1999) (English, with German summary). Dissertation, Technische Universität Berlin, Berlin (1999)

24. Lutz, F.H.: Enumeration and random realization of triangulated surfaces. In: Bobenko, A.I., Schröder, P., Sullivan, J.M., Ziegler, G.M. (eds.) Discrete Differential Geometry. Oberwolfach Seminars, vol. 38, pp. 235-253. Birkhäuser, Basel (2008). Available at arXiv:math.CO/0506316

25. Mahajan, Y.S., Fu, Z., Malik, S.: Zchaff2004: an efficient SAT solver. In: Hoos, H.H., Mitchell, D.G. (eds.) SAT, 2004. Lecture Notes in Computer Science, vol. 3543, pp. 360-375. Springer, Berlin (2005)

26. McMullen, P., Schulz, C., Wills, J.M.: Polyhedral 2-manifolds in $E^{3}$ with unusually large genus. Israel J. Math. 46(1-2), 127-144 (1983)

27. Novik, I.: A note on geometric embeddings of simplicial complexes in a Euclidean space. Discrete Comput. Geom. 23(2), 293-302 (2000)

28. Peirce, C.S.: On the algebra of logic. Am. J. Math. 3, 15-58 (1880)

29. Peterson, B.B.: The geometry of Radon's theorem. Am. Math. Mon. 79, 949-963 (1972)

30. Peyton Jones, S.L. (ed.): Haskell 98 Language and Libraries: The Revised Report. Cambridge University Press, Cambridge (2003)

31. Radon, J.: Mengen konvexer Körper, die einen gemeinsamen Punkt enthalten. Math. Ann. 83, 113115 (1921). (In German)

32. Steinitz, E., Rademacher, H.: Vorlesungen über die Theorie der Polyeder. Springer, Berlin (1934) 
33. Sulanke, T., Lutz, F.H.: Isomorphism free lexicographic enumeration of triangulated surfaces and 3-manifolds. Eur. J. Comb. (2009). doi:10.1016/j.ejc.2008.12.016

34. Timmreck, D.: Necessary conditions for geometric realizability of simplicial complexes. In: Bobenko, A.I., Schröder, P., Sullivan, J.M., Ziegler, G.M. (eds.) Discrete Differential Geometry. Oberwolfach Seminars, vol. 38, pp. 215-233. Birkhäuser, Basel (2008). Available at arXiv:0705.1912

35. Ziegler, G.M.: Polyhedral surfaces of high genus. In: Bobenko, A.I., Schröder, P., Sullivan, J.M., Ziegler, G.M. (eds.) Discrete Differential Geometry. Oberwolfach Seminars, vol. 38, pp. 191-213. Birkhäuser, Basel (2008). Available at arXiv:math.MG/0412093 